\title{
Principais fatores que influenciam a colonização por leveduras do gênero Candida em próteses oculares confeccionadas em resina acrílica
}

\author{
Main factors that influence colonization by yeasts of the genus of Candida in eye prostheses \\ manufactured in acrylic resin
}

Principales factores que influyen en la colonización por levaduras del género de Candida en prótesis oculares fabricadas en resina acrílica

Recebido: 12/04/2021 | Revisado: 20/04/2021 | Aceito: 21/04/2021 | Publicado: 05/05/2021

Kássia Regina de Santana
ORCID: https://orcid.org/0000-0002-4187-5933
Universidade de Pernambuco, Brasil
E-mail: kassia.santana@ @upe.br
Allef Monteiro de Abreu
ORCID: https://orcid.org/0000-0001-6963-6494
Universidade Federal Rural de Pernambuco, Brasil
E-mail: allefmonteiro18.am @gmail.com
Eliana Santos Lyra da Paz
ORCID: https://orcid.org/0000-0003-4486-142X
Universidade de Pernambuco, Brasil
E-mail: eliana.lyra@ upe.br
Emanuel Savio de Souza Andrade
ORCID: https://orcid.org/0000-0003-2165-4217
Universidade de Pernambuco, Brasil
E-mail: emanuel.savio@ upe.br
Francisco Braga da Paz Júnior
ORCID: https://orcid.org/0000-0003-1810-4011
Instituto Federal de Educação, Ciência e Tecnologia de Pernambuco, Brasil
E-mail: franciscobraga@ @recife.ifpe.edu.br
Maria do Socorro Orestes Cardoso
ORCID: https://orcid.org/0000-0001-9866-0899
Universidade de Pernambuco, Brasil
E-mail: socorro.orestes@ upe.br
ORCID: https://orcid.org/0000-0002-9790-8001
Universidade de Pernambuco, Brasil
E-mail: victor.prado@upe.br

\begin{abstract}
Resumo
Esta pesquisa trata-se de uma revisão integrativa, com o objetivo de demostrar os principais fatores que influenciam a colonização por leveduras do gênero Candida em próteses oculares confeccionadas em resina acrílica e a composição microbiológica da secreção conjuntival presente nas cavidades anoftálmicas. Foi realizada a recuperação de estudos publicados, através de um levantamento bibliográfico por meio de estratégia de busca com base nos termos: Candida; Fatores de virulência; Infecções oculares fúngicas; Olho artificial. Sendo os critérios de inclusão: artigos publicados em português, inglês e espanhol; artigos indexados nas bases de dados: Literatura Latino-Americana e do Caribe em Ciências da Saúde (LILACS), National Library Of Medicine Online (MEDLINE), Scopus e Scientific Electronic Library Online (SciELO); artigos publicados no período de 2001 a 2020. E, como critérios de exclusão: estudos sem informações sobre a amostragem e análise efetuada; estudos de revisão bibliográfica e estudos com duplicidades nas bases de dados. Dentre os fatores que influenciam a colonização das póteses oculares por leveduras do gênero Candida, se destacam: Qualidade do acabamento e polimento das próteses; grau de porosidades e rugosidades superficiais; qualidade da resina acrílica empregada na confecção e grau de conversão dos polímeros; frequência de higienização da prótese e da mucosa conjuntival; secreção lacrimal da cavidade anolftálmica; e fatores de virulência das leveduras colonizadoras. Dessa forma, ressaltamos que o controle da carga microbiana da cavidade anoftálmica,
\end{abstract}


através do emprego correto da técnica de confecção e boa prática de higienização, mostra-se imprescindível para que a reabilitação através do uso das próteses oculares desempenhe seu papel de forma exitosa.

Palavras-chave: Candida; Fatores de virulência; Infecções oculares fúngicas; Olho artificial.

\begin{abstract}
This research was an integrative review, with the objective of demonstrate the main factors that influence colonization by Candida yeasts in ocular prosthesis manufactured of acrylic resin and a microbiological composition of conjunctival secretion present in anophthalmic cavities. Published studies were retrieved through a bibliographic survey using a search strategy based on the terms: Candida; Virulence Factors; Eye Infections, Fungal; Eye, Artificial. with Inclusion criteria: articles published in Portuguese, English and Spanish; articles indexed in the databases: Latin American and Caribbean Literature in Health Sciences (LILACS), National Library Of Medicine Online (MEDLINE), Scopus and Scientific Electronic Library Online (SciELO); articles published from 2001 to 2020. And, as exclusion criteria: studies without information on sampling and analysis performed; bibliographic review studies and studies with duplicates in the databases. Among the factors that influence the colonization of ocular prostheses by Candida yeasts, the following stand out: Quality of the finishing and polishing of the prostheses; degree of surface porosity and roughness; quality of the acrylic resin used in the manufacture and degree of conversion of the polymers; frequency of cleaning of the prosthesis and conjunctival mucosa; lacrimal secretion from the anophthalmic cavity; and virulence factors of colonizing yeasts. Thus, we emphasize that the control of the microbial load of the anophthalmic cavity, through the correct use of the confection technique and good hygiene practice, is essential for rehabilitation through the use of ocular prostheses to play its role successfully.
\end{abstract}

Keywords: Candida; Virulence Factors; Eye Infections, Fungal; Eye, Artificial.

\title{
Resumen
}

Esta investigación es una revisión integradora, con el objetivo de demostrar los principales factores que influyen en la colonización por levaduras del género Candida en prótesis oculares de resina acrílica y la composición microbiológica de la secreción conjuntival presente en cavidades anoftálmicas. Los estudios publicados se recuperaron a través de una encuesta bibliográfica utilizando una estrategia de búsqueda basada en los términos: Candida; Factores de Virulencia; Infecciones Fúngicas del Ojo; Ojo Artificial. Criterios de inclusión: artículos publicados en portugués, inglés y español; artículos indexados en las bases de datos: Literatura Latinoamericana y del Caribe en Ciencias de la Salud (LILACS), Biblioteca Nacional de Medicina en Línea (MEDLINE), Scopus y Biblioteca Electrónica Científica en Línea (SciELO); artículos publicados entre 2001 y 2020. Y, como criterio de exclusión: estudios sin información sobre muestreo y análisis realizados; estudios de revisión bibliográfica y estudios con duplicados en las bases de datos. Entre los factores que influyen en la colonización de prótesis oculares por levaduras Candida, destacan: Calidad del acabado y pulido de las prótesis; grado de porosidad y rugosidad de la superficie; calidad de la resina acrílica utilizada en la fabricación y grado de conversión de los polímeros; frecuencia de limpieza de la prótesis y mucosa conjuntival; secreción lagrimal de la cavidad anoftálmica; y factores de virulencia de las levaduras colonizadoras. Así, destacamos que el control de la carga microbiana de la cavidad anoftálmica, mediante el uso correcto de la técnica de cocción y buenas prácticas de higiene, es fundamental para que la rehabilitación mediante el uso de prótesis oculares desempeñe su función con éxito.

Palabras clave: Candida; Factores de Virulencia; Infecciones Fúngicas del Ojo; Ojo Artificial.

\section{Introdução}

A utilização das próteses oculares é disposta de forma semelhante por pacientes com diferentes idades (Modugno, et al., 2013), essa disposição é fundamentada na etiologia diversificada das amputações oculares, onde podemos destacar os tumores, defeitos congênitos, malformações, traumas irreparáveis, doenças oculares em estágio final, doenças oculares graves que levam à cegueira associada à dor descontrolada ou aparência estética desagradável, como infecções intraoculares ou complicações pós-cirúrgicas (Artopoulou, et al., 2006; Modugno, et al., 2013; Zhang, et al., 2015).

$\mathrm{O}$ tratamento reabilitador através do uso de próteses oculares é indicado diante de perdas totais ou parciais do globo ocular (Bento, et al., 2018), pois, mesmo não restituindo a principal função do globo ocular, a visão, as mesmas são capazes de alcançar bons níveis de reestabelecimento da harmonia facial, autoestima do paciente, de proteção da mucosa adjacente da cavidade anoftálmica, orientação do caminho da secreção lacrimal e auxilio na proteção contra infecções, irritações e inflamações (Carvalho, Piass \& Haddad, 2020).

O olho humano encontra-se constantemente passível a problemas decorrentes de infecções, seja por desarmonia na microbiota residente ou pela exposição a microrganismos exógenos, assim como pela deficiência do sistema imune ocular 
(Souza, 2018). Sendo a suscetibilidade a infecções ampliada diante da perda do globo ocular, visto que o mesmo representa barreira de proteção para conjuntiva local.

Com o aumento da suscetibilidade da mucosa constituinte da cavidade anoftálmica, após a perda ocular, através de situações de imunossupressão ou alterações sistêmicas que provoquem mudanças nas características físico-químicas do hospedeiro (da Silva, Vieira \& de Luna Gurgel, 2019), alguns microrganismos residentes, oportunistas, assumem um comportamento patogênico. Esse é o caso das manifestações infecciosas causadas por Candida.

Diante do risco de agravos decorrentes de microrganismos oportunistas, em especial das leveduras do gênero Candida, torna-se imprescindível o controle do biofilme para manutenção da saúde da cavidade anoftálmica dos portadores de próteses oculares, visto que as propriedades inerentes da resina acrílica, como a rugosidade superficial, podem favorecer a adesão de microrganismos nas próteses (Andreotti, 2017). Dessa forma, a adoção de métodos que inativem os mesmos da sua superfície ou que desorganizem o biofilme, demonstra sua importância para prevenção e tratamento de infecções (Tasso, 2019).

A principal forma de inativação e desorganização do biofilme é através da boa higienização, tanto da prótese quanto da mucosa adjacente. Evelin (2019), destacou como principais procedimentos antissépticos a higienização das próteses com sabão ou xampu neutro, clorexidina e soluções para lentes de contato, devendo ser evitado sabão inespecífico ou produtos que contenham álcool ou abrasivos, uma vez que podem causar danos às próteses e aos tecidos remanescentes da cavidade anoftálmica.

Em face das considerações descritas, esta revisão integrativa objetivou demostrar a importância de estudos visando à compreensão dos principais fatores que influenciam a colonização por leveduras do gênero Candida em próteses oculares confeccionadas em resina acrílica e a composição microbiológica da secreção conjuntival presente nas cavidades anoftálmicas, com propósito de entender melhor a fisiopatologia das infecções neste sítio.

\section{Metodologia}

Esta pesquisa trata-se de uma revisão de literatura, através do método qualitativo para análise dos dados (Pereira, et al., 2018). Foi realizada a recuperação de estudos publicados sobre a colonização por leveduras do gênero Candida em próteses oculares confeccionadas em resina acrílica, através de um levantamento bibliográfico por meio de estratégia de busca com base nos termos: Candida, Fatores de Virulência, Infecções Oculares Fúngicas, Olho Artificial. Os resumos dos artigos recuperados foram analisados para verificar o atendimento aos critérios de inclusão e exclusão. Sendo os critérios de inclusão: artigos publicados em português, inglês e espanhol; artigos indexados nas bases de dados Literatura Latino-Americana e do Caribe em Ciências da Saúde (LILACS), National Library Of Medicine Online (MEDLINE), Scopus e Scientific Electronic Library Online (SciELO); artigos publicados no período de 2001 a 2020. E, como critérios de exclusão: estudos sem informações sobre a amostragem e análise efetuada; estudos de revisão bibliográfica e estudos com duplicidades nas bases bibliográficas.

As buscas foram realizadas na seção de busca dos sites, abrangendo a pesquisa aos títulos, resumos e palavras-chave dos artigos. Todos os registros de artigos publicados entre 1998 e 2020, que continham qualquer um desses termos em qualquer um dos campos da base de dados (título, resumo ou palavras-chave), foram identificados, armazenados e tratados.

Para explorar e organizar as informações contidas nos artigos selecionados válidos, os dados foram armazenados e tabulados em planilhas compatíveis com o software Microsoft Excel 2010. O recorte temporal de 22 anos foi escolhido para abranger um período de estudo sobre a temática, a fim de garantir a relevância e atualidade desta pesquisa. 


\section{Resultados e Discussão}

\subsection{Próteses oculares}

A prótese ocular integra uma modalidade de reabilitação indicada em casos de perda ocular, parcial ou total. Essa reabilitação é considerada um desafio, com alto nível de complexidade e requer grande investimento de mão de obra do operador. Isto porque possui uma relevância diretamente ligada à qualidade de vida, recuperação da autoconfiança, além de interferir no convívio social dos pacientes (Rodrigues, Rodrigues \& Oliveira, 2019).

De acordo com Gonçalves et al. (2018), a reabilitação do paciente pode ser pelo uso de próteses oculares individualizadas ou próteses pré-fabricadas. Diferente das próteses pré-fabricadas, que são de estoque e nem sempre conseguem restabelecer a estética e a movimentação de maneira satisfatória, a prótese ocular individualizada (Figura 1,2) é feita artesanalmente e é ajustada à estrutura ocular do paciente, sendo mais propensas a mimetizarem os movimentos e a estética ocular.

Figura 1 - Esclera caracterizada e íris artificial colada sobre o platô

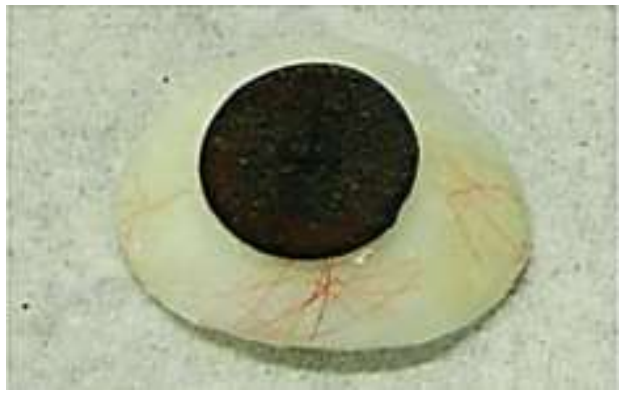

Fonte: Bento et al. (2018).

O material mais utilizado na confecção das próteses oculares são as resinas acrílicas termicamente ativadas. Goiato et al. (2012), destacaram como principais características desse insumo a biocompatibilidade, fácil acesso e manipulação, estética satisfatória, e favorável para o paciente e para o cirurgião-dentista. Porém, por suas características inerentes, as resinas acrílicas exigem cuidados específicos para conservação da prótese e manutenção da saúde dos tecidos remanescentes (Marques, et al., 2018).

Figura 2 - a) Aspecto inicial do paciente anoftálmico do lado esquerdo. b) Aspecto final, após a instalação da prótese ocular.

a)

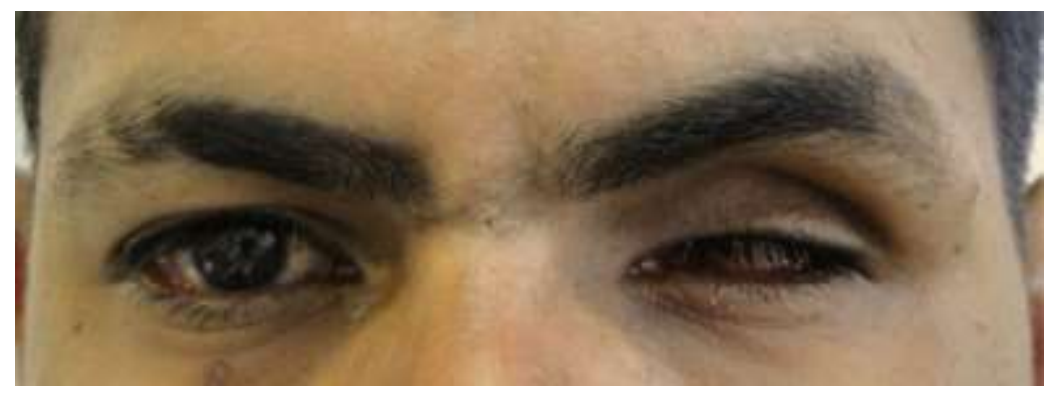


b)

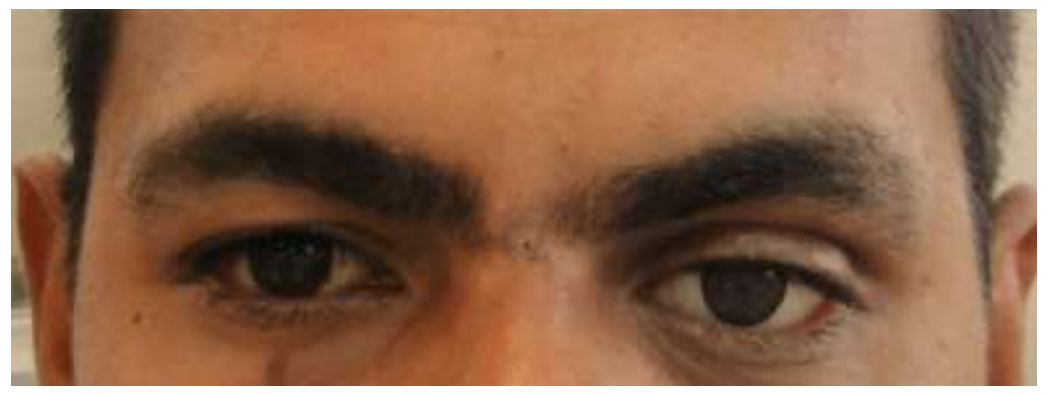

Fonte: Carvalho, Piass \& Haddad (2020).

\subsubsection{Próteses oculares e a colonização microbiana}

Segundo Tavares et al. (2018), a colonização da cavidade anoftálmica está associada às características inerentes do indivíduo portador da prótese, com o grau de porosidade e rugosidade da resina acrílica utilizada na confecção. O favorecimento da colonização microbiana também advém de lesões traumáticas causadas por próteses mal adaptadas (Falcão, Santos \& Sampaio, 2004).

Em se tratando dos fatores de suscetibilidade à colonização microbiana ligados a prótese, também pode ser mencionado o espaço formado entre a superfície posterior da prótese e cavidade anoftálmica. Esse espaço pode ser observado mesmo nas próteses individualizadas, onde a moldagem é feita diretamente na cavidade anoftálmica. O acúmulo de lágrima e muco é observado nesse espaço, formando um meio nutritivo, que favorece o crescimento microbiano e aumenta a vulnerabilidade do paciente ao desenvolvimento de patologias locais (Figura 3), como conjuntivite crônica de superfície, por exemplo (Cardoso, Carneiro, Vasconcelos \& Melo, 2011).

Figura 3 - Fotos contínuas em escala de classificação usada com interpolação para medir a gravidade da inflamação na conjuntiva palpebral.
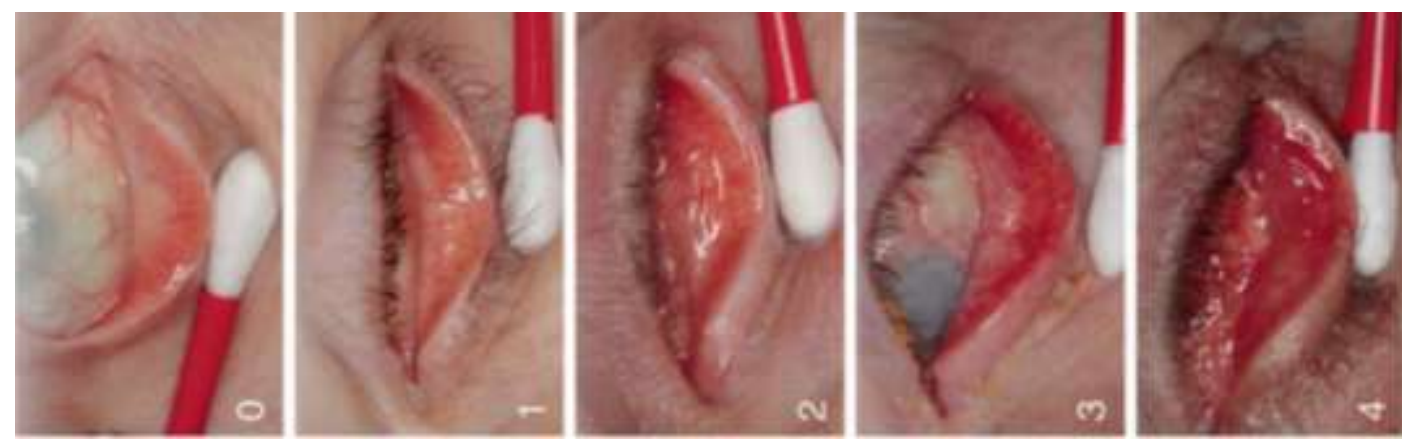

Fonte: Pine et al. (2013).

\subsubsection{Higienização da prótese ocular e controle do biofilme}

Segundo Paranhos et al. (2007), a má conservação e a ausência de higienização da prótese podem provocar irritação, bem como inflamação da mucosa de revestimento da cavidade anoftálmica.

Cuidados diários quanto a sua higienização devem ser tomados. Rezeck et al. (2020), construíram um manual de cuidados e higienização para usuários de prótese ocular, onde foi ressaltada a importância da lavagem das mãos e vedamento da pia antes da higienização das próteses oculares, as quais devem ser limpas diariamente com água e sabão neutro, nunca com álcool ou qualquer produto corrosivo, o que danificaria o material. É permitido que o paciente durma com a prótese, entretanto, 
se ele não o quiser, deverá mantê-la acondicionada em uma gaze umedecida com água. Para melhorar a lubrificação local, deve-se depositar no interior da cavidade anoftálmica, uma gota de óleo mineral, duas vezes ao dia.

No que concerne aos cuidados semanais (Figura 4), deve ser procedida a desinfecção da prótese ocular, através da imersão do mesmo em peróxido de hidrogênio a 10 volumes, por 10 minutos ou a 20 volumes, por 5 minutos. Logo em seguida, deve ser realizada a limpeza com soro fisiológico estéril para remoção de resíduos do desinfetante (Rezeck, et al., 2020). Dentre os cuidados a longo prazo, Rezeck et al. (2020), recomenda o polimento a cada 6 meses e a troca da prótese a cada 5 anos, o que poderá garantir a qualidade do material e conforto ao paciente.

Figura 4 - Manual de cuidados e higienização para usuários de prótese ocular.

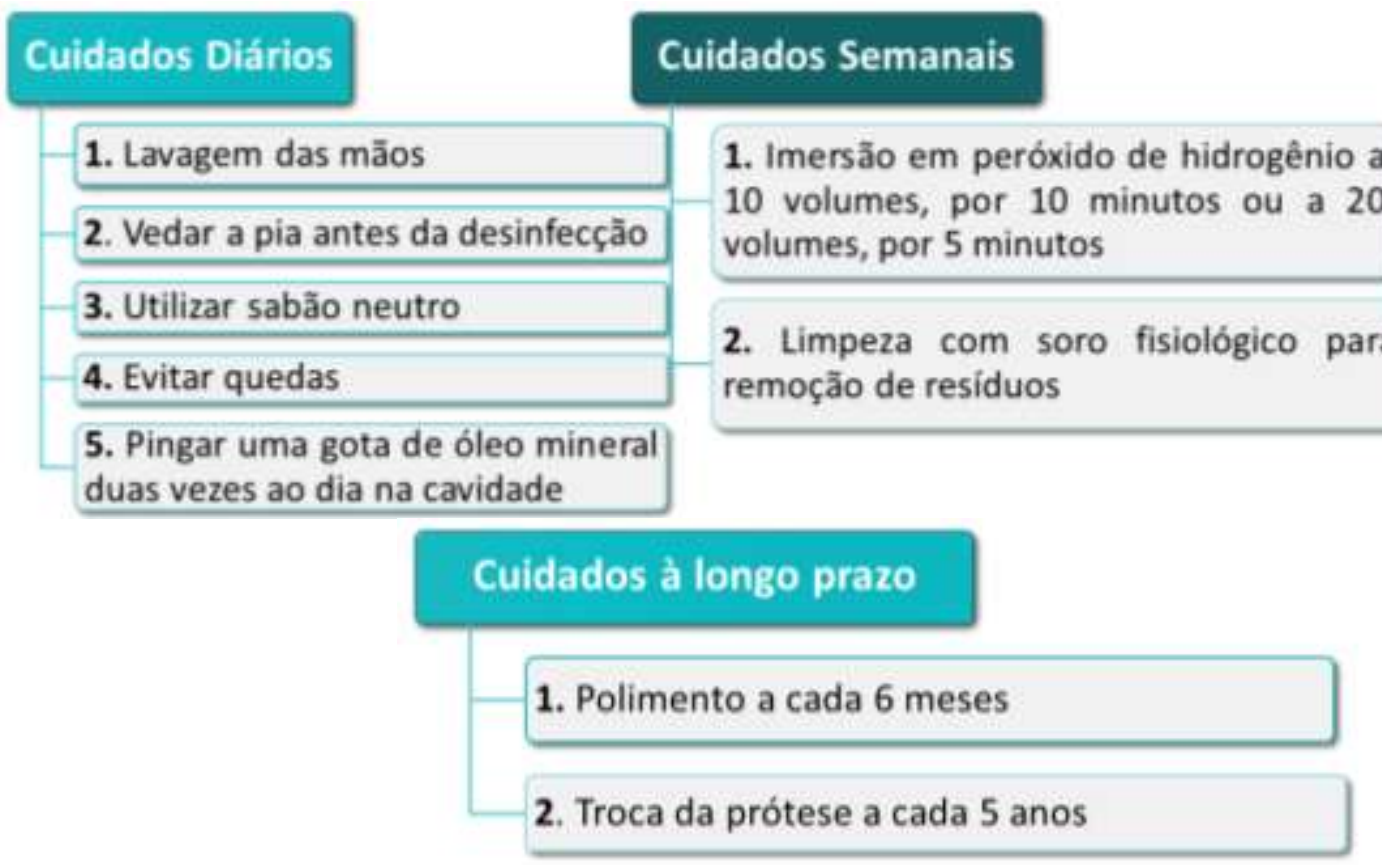

Fonte: Rezeck et al. (2020).

Raizada \& Rani (2007), realizaram um estudo salientando as vantagens do uso de próteses oculares personalizadas, desde que seguidas as orientações para manuseamento e higiene. Os autores sinalizaram que secreções mucosas excessivas podem acontecer quando se utiliza uma prótese ocular. A quantidade média de higienização recomendada neste estudo é de 3 a 5 vezes por dia e toda vez que a prótese for removida da cavidade, deve ser limpa antes da inserção. Nunca limpar a prótese com pano, sabão abrasivo ou pasta de dente e sim com sabão neutro ou shampoo para bebês, devendo ser completamente removido em água corrente antes da reinserção da prótese na cavidade.

\subsubsection{Fatores que influenciam a vida útil da prótese}

A porosidade e rugosidade da superfície das próteses oculares confeccionadas em resina acrílica estão diretamente relacionadas com sua vida útil, assim como com o grau de aderência microbiana. Segundo Raizada \& Rani (2007), a exposição a variações de temperatura, degradação pelo tempo de uso, alterações causadas por soluções e métodos de limpeza e quedas são fatores que podem alterar a rugosidade superficial das próteses oculares. Além disso, as porosidades associadas a falhas na confecção (Canadas, et al., 2010) e no polimento final da prótese (Braun, et al., 2003), que comprometem o grau de rugosidade superficial. 
Em estudo, Osborn \& Hetler (2010), avaliaram uma série de orientações dadas aos pacientes usuários de próteses oculares, buscando avaliar a possibilidade de padronizar as recomendações. Para algumas perguntas, houve mais de uma resposta para incluir as necessidades individuais dos pacientes. Segundo as respostas, o motivo mais frequentemente relatado pelos usuários para remoção da prótese é irritação ou desconforto (31\%); 58\% indicaram a limpeza da prótese sempre que for removida sendo que sabonete neutro e shampoo de bebê foram listados como os agentes de limpeza mais utilizados (24\%); $29 \%$ dos entrevistados citaram que é comum o uso de lubrificante à base de óleo de silicone. Os autores concluíram que não existe um método que funcione para todos e que um plano de tratamento individualizado deve ser realizado para a maioria dos indivíduos.

\subsubsection{Influências das condições da prótese para a mucosa conjuntival}

Pine, Sloan \& Jacobs (2013), realizaram um estudo objetivando o desenvolvolvimento de instrumentos para mensurar a condição das próteses oculares e a resposta da cavidade à prótese ocular. Foi desenvolvida uma nova técnica de coloração para exibir depósitos em olhos protéticos. Indicando que a interface entre a conjuntiva da cavidade anolftálmica e a prótese é responsável pelo maior acúmulo de microrganismos. Os autores salientaram que a técnica para coloração de depósitos e as escalas para medir sua intensidade e extensão atendem às necessidades clínicas e podem auxiliar futuras investigações sobre o desgaste da prótese e afirmam ainda que a escala para medir a inflamação conjuntival é provavelmente útil para a prática clínica, pois permite aos profissionais monitorar o nível de inflamação conjuntival em cavidades anoftálmicas.

O material utilizado na confecção da prótese ocular está intrinsecamente ligado à resposta da mucosa conjuntival durante sua utilização. Por suas características já mencionadas nesse estudo, a resina acrílica é o insumo de escolha. Porém, outros insumos, como o vidro, são usados. Sperb, Neves \& Rode (2001), caracterizaram as próteses de vidro como: De confecção extremamente trabalhosa, padronizadas quanto à forma e à cor, mobilidade reduzida, quando atacada pelo fluoreto existente na lágrima, toma-se opaca e áspera, havendo necessidade de substituição da prótese, em média, a cada dois anos, onde a utilização de próteses confeccionadas com esse material não foram recomendadas por esse autor.

Desde o advento da resina acrílica, as próteses de vidro, tiveram seu espaço cada vez mais restrito. Para analisar as diferenças das preocupações dos usuários de próteses oculares confeccionadas em resina acrílica e vidro (criolita), Rokohl, et al. (2018), realizaram um estudo, onde o lacrimejamento, a formação de crostas e a secreção foram as manifestações específicas mais comuns no grupo da prótese de vidro criolita, sem diferença significativa com a prótese em resina acrílica $(\mathrm{p}=0,667)$. Um alto porcentual de pacientes nos grupos vidro criolita $(88,2 \%)$ e resina acrílica $(91,2 \%)$ demostraram lacrimejamento, formação de crostas e secreção, diariamente. Quando os participantes foram solicitados a indicar a condição que mais os preocupavam entre lacrimejamento, formação de crosta e secreção ou aparência, $61,8 \%$ do grupo de vidro criolita e $58,8 \%$ do grupo de resina acrílica marcaram as três complicações.

\subsection{Microbiota da cavidade anoftálmica}

A microbiota oportunista residente da cavidade ocular, como as leveduras do gênero Candida, por exemplo, em situações onde há quebra na homeostase, está associada com o desenvolvimento de patologias locais. Fatores como o uso de medicamentos imunossupressores, uso indiscriminado de antibióticos e corticoides, em associação com aspectos ambientais relacionados à exposição ocupacional ao meio rural, principalmente no que se refere aos fungos (de Souza, et al. 2020).

Avaliando a relação entre a microbiota conjuntival e conforto da cavidade em indivíduos anoftálmicos, Toribio et al. (2017) observaram que o crescimento da microbiota patogênica, especialmente bactérias Gram negativas e o aumento da densidade da microbiota estão relacionados ao desconforto. Ainda Pinheiro et al. (2018), avaliaram que a microbiota da cavidade anoftálmica e a prótese ocular de 26 indivíduos, por meio da técnica de DNA-checkerboard, identificaram a presença 
de 38 espécies diferentes de microrganismos colonizando ambos os sítios. Estes relatos indicam, portanto, a necessidade do estabelecimento de protocolos eficientes para controle da microbiota.

Para de Souza et al. (2020), os microrganismos envolvidos com maior frequência nas infecções em usuários de próteses oculares são bactérias e fungos, sendo algumas bactérias advindas da microbiota ocular como espécies de Staphylococcus e Corynebacterium e outras com características estritamente patogênicas como espécies de Streptococcus, e Pseudomonas. Diferente do que acontece com os fungos, que são encontrados no ambiente ocular em baixa frequência de maneira constitutiva da microbiota comum, porém em situações de desequilíbrio ocasionam patologias oculares. Os espécimes fúngicos mais frequentes foram: Fusarium spp (90,5\%) e Aspergillus spp. (9,5\%) dentre as 50 culturas positivas analisadas.

Toribio, et al. (2017), realizaram um estudo clínico buscando verificar as possíveis diferenças e relações entre as microbiotas dos diferentes espaços conjuntivais, relacionando os achados ao conforto do paciente. Sessenta pacientes com anoftalmia unilateral e usuários de prótese ocular foram convidados a participar. Os resultados indicaram menor densidade microbiana nos olhos saudáveis quando comparado com demais amostras. Verificou-se que houve correlação entre a densidade microbiana dos três sítios coletados e o baixo índice de conforto relatado. A presença de bactérias aeróbias Gram negativas nas amostras colatadas na região posterior da prótese de pacientes com conforto ruim foi maior que em pacientes com grau de conforto bom. Os autores concluíram que o grau de conforto da cavidade anoftálmica está relacionado à miocrobiota.

A perda do globo ocular, através da exposição da conjuntiva da cavidade anolfitálmica é responsável por quadros de diminuição da secreção lacrima, aumentando assim, os riscos de problemas decorrentes da colonização e infecção, visto que, a superfície ocular e da mucosa são protegidos pela secreção lacrimal que impede a penetração de alguns microrganismos potencialmente patogénicos. A lágrima integra uma enorme diversidade de moléculas antimicrobianas, como lisozima, lactoferrina e lipocalina, que auxiliam na citólise e impossibilitam o desenvolvimento dos tais microrganismos patogénicos (Carvalho, 2020).

\subsection{Leveduras do gênero Candida}

As espécies do gênero Candida são as mais comumente associadas às infecções fúngicas em seres humanos (Pais, et al. 2019). Segundo Nunes (2019), a Candida albicans é o principal fungo patógeno humano, responsável por infecções. Sua prevalência está fortemente associada com a formação de biofilmes.

De acordo com de Souza Paz et al. (2019), as infecções geralmente ocorrem quando há uma ruptura do equilíbrio biológico, resultante de fatores fisiológicos, imunológicos, mecânicos ou patológicos. Segundo Ataídes et al. (2010), alterações do equilíbrio, devido ao acréscimo e/ou disseminação de leveduras no tecido e instalação da infecção podem manifestar quadros clínicos benignos, como infecções de mucosas e pele, ou quadros graves e fatais como as candidemias (infecções disseminadas).

\subsubsection{Candida Albicans}

A Candida albicans é considerado o principal patógeno fúngico humano, responsável por doenças que vão desde a mucosa superficial até infecções sistêmicas com risco de vida (Tsui, Kong \& Jabra-Rizk, 2016). Esta levedura é habitualmente isolada na microbiota normal de variados locais anatômicos. 
Figura 5 - Aspectos ultraestruturais de C. albicans: A) forma oval (levedura) e B) forma filamentosa.

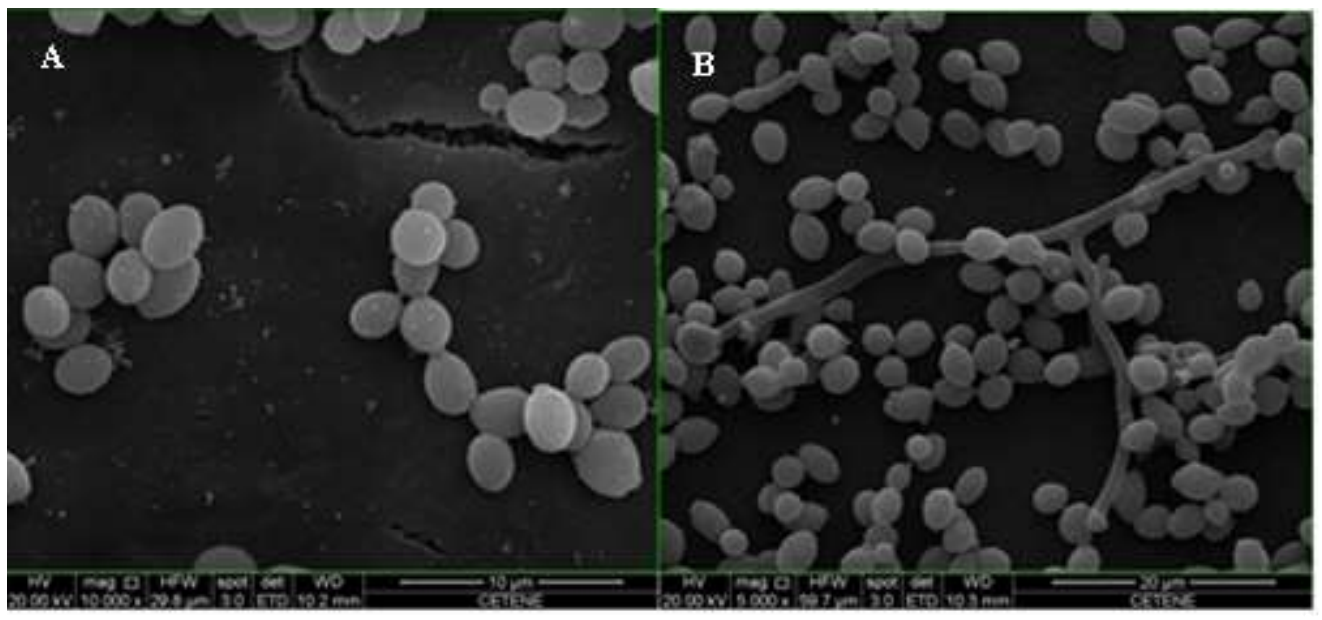

Fonte: Cavalcanti Filho et al (2017).

Para Cavalcanti Filho et al. (2017), vários fatores, relacionados ao hospedeiro e ao patógeno, são reconhecidos como cruciais para o desenvolvimento da infecção por C. albicans, que possui uma gama de fatores de virulência, associados à aderência, filamentação, formação de biofilme e secreção de proteases.

Zanelatto (2015) em seu estudo concluiu que geralmente $50 \%$ dos indivíduos são portadores de C. albicans em sua forma comensal. Os termos Candidíase ou Candidose referem-se ao processo infeccioso causado pelos fungos do gênero Candida e as manifestações da doença variam de acordo com o sítio anatômico acometido (Avrella \& Goulart, 2008; de Melo \& Guerra, 2014; da Paz, et al. 2020).

\section{Considerações Finais}

Dentre os fatores que influenciam a colonização das próteses oculares por leveduras do gênero Candida, se destacam: Qualidade do acabamento e polimento das próteses; grau de porosidades e rugosidades superficiais; qualidade da resina acrílica empregada na confecção e grau de conversão dos polímeros; frequência de higienização da prótese e da mucosa conjuntival; secreção lacrimal da cavidade anolftálmica; e fatores de virulência das leveduras colonizadoras.

Dessa forma, ressaltamos que o controle da carga microbiana da cavidade anoftálmica, através do emprego correto da técnica de confecção e boa prática de higienização, mostra-se imprescindível para que a reabilitação através do uso das próteses oculares desempenhe seu papel de forma exitosa.

Diante dessas considerações, e observando que a resina acrílica utilizada na confecção das próteses oculares são fontes iminentes de acúmulo de microrganismos, torna-se evidente a importância de estudos futuros a respeito dos fatores que aumentam a suscetibilidade da prótese ocular à adesão microbiana e a vulnerabilidade do hospedeiro à infecção por leveduras do gênero Candida.

\section{Referências}

Andreotti, A. M. (2017). Influência da rugosidade de superfície e ação de diferentes desinfetantes na formação de biofilme em resina acrílica para prótese ocular e sua biocompatibilidade em contato com células da conjuntiva humana. Trabalho de Conclusão de Curso (TCC). Repositório Institucional.

Artopoulou, I. I., Montgomery, P. C., Wesley, P. J., \& Lemon, J. C. (2006). Digital imaging in the fabrication of ocular prostheses. The Journal of prosthetic dentistry, 95(4), 327-330. https://doi.org/10.1016/j.prosdent.2006.01.018.

Ataídes, F. S., Abrão, F. Y., Costa, C. R., Silva, M. D. R. R., Pimenta, F. C., Prado-Palos, M. A., \& Souza, L. K. H. (2010). Identificação de espécies de Candida em saliva de profissionais da saúde. Revista Eletrônica de Enfermagem, 12 (3), 498-501. https://doi.org/10.5216/ree.v12i3.6472.

Avrella, D., \& Goulart, L. S. G. (2008). Isolamento de Candida spp. da mucosa oral de pacientes submetidos ao tratamento quimioterápico. Rev. Bras. Anal. Clin, 40 (3), 205-207. 
Bento, G., Marinho, L. R., Reis, M. S. D., \& Haddad, M. F. (2018). Reabilitação de paciente anoftálmico bilateral por meio de prótese ocular: relato de caso. Arch. Health Invest, 7 (10), 446-449. https://doi.org/10.21270/archi.v7i10.3028.

Braun, K. O. N., Mello, J. A. N., Rached, R., \& Del Bel Cury, A. A. (2003). Surface texture and some properties of acrylic resins submitted to chemical polishing. Journal of oral rehabilitation, 30 (1), 91-98. https://doi.org/10.1046/j.1365-2842.2003.00997.x..

Canadas, M. D. B., Garcia, L. F. R., Consani, S., \& Pires-de-Souza, F. C. P. (2010). Color stability, surface roughness, and surface porosity of acrylic resins for eye sclera polymerized by different heat sources. Journal of Prosthodontics: Implant, Esthetic and Reconstructive Dentistry, 19(1), 52-57. https://doi.org/10.1111/j.1532-849X.2009.00522.x.

Cardoso, M. D. S. O., Carneiro, S. C. D. A. S., Vasconcelos, B. C. D. E., \& Melo, A. R. (2011). Microbiota da Secreção Conjuntival em Portadores de Prótese Ocular. Revista de Cirurgia e Traumatologia Buco-maxilo-facial, 11(2), 109-114.

Carvalho, A. C. D. (2020). Aconselhamento farmacêutico especializado em saúde ocular: olho seco. Tese de Doutorado. Repositório Institucional.

Carvalho, B. A., Piassi, J. E. V., \& Haddad, M. F. (2020). Tratamento reabilitador de deformidade ocular: relato de caso. Rev. Odontol. Araçatuba, 41 (1), 1923.

Cavalcanti Filho, J. R. N., Silva, T. F., Nobre, W. Q., Oliveira de Souza, L. I., Silva e Silva Figueiredo, C. S., Figueiredo, R. C. B. Q. D., Gusmão, N. B. D., Silva, M. V., Silva, Luís C. N. D., \& Correia, M. T. D. S. (2017). Antimicrobial activity of Buchenavia tetraphylla against Candida albicans strains isolated from vaginal secretions. Pharmaceutical biology, 55(1), 1521-1527. https://doi.org/10.1080/13880209.2017.1304427.

da Paz, E. S. L., de Abreu, A. M., Mandú, A. L. C., de Lira, C. R. S., Guaraná, C. F. R., da Paz Júnior, F.B., Alves, J., de Santana, K. R., Barbosa, L. M., \& de Oliveira Cavalcanti, M. T. M. (2020). Prevalência de Candida em próteses dentárias de pacientes oriundos das clínicas da Faculdade de Odontologia de Pernambuco. Research, Society and Development, 9(9), 970998011-970998011. https://doi.org/10.33448/rsd-v9i9.8011.

da Silva, E. F., Vieira, T. J. N., \& de Luna Gurgel, G. C. D. (2019). Atividade biológica" in vitro" do fitoconstituinte timol sobre espécies do gênero Candida. Revista de Pesquisa Interdisciplinar, 2(2.0). http://dx.doi.org/10.24219/rpi.v2i2.0.396.

de Melo, I. A., \& Guerra, R. C. (2014). Candidíase oral: um enfoque sobre a estomatite por prótese. Salusvita, 33 (3), 389-414.

de Souza, M. S, Júnior, A. C. V., de Oliveira, D. C., da Silva Guedes, D. R., de Macedo, C. A., Araújo, G. M., Fernandes, J. V., \& Andrade, V. S. (2020). Perfil microbiano de infecções oculares em pacientes atendidos no hospital universitário do estado do Rio Grande Do Norte. Brazilian Journal of Development, 6(4), 19758-19775. https://10.34117/bjdv6n4-226.

de Souza Paz, C. Q., Teixeira, A. C. O. C., Jacobi, C. C. B., Santos, F. F. C., dos Santos Santana, H. C. P., \& Borges-Paluch, L. R. (2019). Riscos associados à colonização por Candida na cavidade oral de escolares da Rede Municipal de Governador Mangabeira-BA. Revista da Universidade Vale do Rio Verde, 17(1). http://dx.doi.org/10.5892/ruvrd.v17i1.4491.

Evelin, L. D. S. (2019). Influência de dois protocolos de higiene para usuários de prótese ocular na condição de saúde dos tecidos, microbiota da cavidade anoftálmica e nível de satisfação dos pacientes. Tese de Doutorado. Repositório Institucional.

Falcão, A. F. P., Santos, L. D. B., \& Sampaio, N. D. M. (2004). Candidíase associada a próteses dentárias. Sitientibus, 30(1), 135-46.

Goiato, M. C., dos Santos, D. M., Moreno, A., Iyda, M. G., Rezende, M. C., \& Haddad, M. F. (2012). Effect of disinfection and storage on the flexural strength of ocular prosthetic acrylic resins. Gerodontology, 29(2), 838-844.

Gonçalves, K. V., Martins, O. B. T., Neves, M. D. S., \& Haddad, M. F. (2018). Reabilitação de paciente eviscerado por meio de prótese ocular: relato de caso. Arch. Health Invest, 7 (8), 329-333. http://dx.doi.org/10.21270/archi.v7i8.3027.

Marques, M.C., Caxias, F. P., Santos, D. M., Telles, K. L., Ervolino, I. C. S., Souza, J. P. V., Sousa M. V. P., \& Goiato, M. C. (2018). Qualidade de vida de usuários de prótese ocular. Archives Of Health Investigation, 7. http://dx.doi.org/10.21270/archi.v7i0.3910.

Modugno, A., Mantelli, F., Sposato, S., Moretti, C., Lambiase, A., \& Bonini, S. (2013). Ocular prostheses in the last century: a retrospective analysis of 8018 patients. Eye, 27(7), 865-870. http://dx.doi.org/10.1038/eye.2013.97.

Nunes, T. S. B. S. (2019). Efeito da N-acetilcisteína em biofilme de Candida albicans. Trabalho de conclusão de curso (TCC). Repositório Institucional.

Osborn, K. L., \& Hettler, D. (2010). A survey of recommendations on the care of ocular prostheses. Optometry-Journal of the American Optometric Association, 81(3), 142-145. https://doi.org/10.1016/j.optm.2009.11.003.

Pais, P., Galocha, M., Viana, R., Cavalheiro, M., Pereira, D., \& Teixeira, M. C. (2019). Microevolution of the pathogenic yeasts Candida albicans and Candida glabrata during antifungal therapy and host infection. Microbial Cell, 6(3), 142. https://doi.org/10.15698/mic2019.03.670.

Paranhos, R. M. Z. F., Batalhão, C. H., Semprini, M., Regalo, S. C. H., Ito, I. Y., \& Mattos, M. D. G. C. D. (2007). Evaluation of ocular prosthesis biofilm and anophthalmic cavity contamination after use of three cleansing solutions. Journal of Applied Oral Science, 15(1), 33-38. http://dx.doi.org/10.1590/S167877572007000100008 .

Pereira, A. S., Shitsuka, D. M., Parreira, F. J., \& Shitsuka, R. (2018). Metodologia da pesquisa científica. E-book.

Pine, K. R., Sloan, B., \& Jacobs, R. J. (2013). The development of measurement tools for prosthetic eye research. Clinical and Experimental Optometry, 96(1), 32-38. https://doi.org/10.1111/j.1444-0938.2012.00754.x.

Pine, K. R., Sloan, B., Stewart, J., \& Jacobs, R. J. (2013). The response of the anophthalmic socket to prosthetic eye wear. Clinical and Experimental Optometry, 96(4), 388-393. https://doi.org/10.1111/cxo.12004. 
Research, Society and Development, v. 10, n. 5, e27210514990, 2021

(CC BY 4.0) | ISSN 2525-3409 | DOI: http://dx.doi.org/10.33448/rsd-v10i5.14990

Pinheiro, J. B., Vomero, M. P., do Nascimento, C., Watanabe, E., Paranhos, H. D. F. O., Coto, N. P., Dias, R. B., de Oliveira, V. C., \& Silva-Lovato, C. H. (2018). Genomic identification of microbial species adhering to maxillofacial prostheses and susceptibility to different hygiene protocols. Biofouling, 34(1), 15-25. https://doi.org/10.1080/08927014.2017.1403591

Raizada, K., \& Rani, D. (2007). Ocular prosthesis. Contact Lens and Anterior Eye, 30(3), 152-162. https://doi.org/10.1016/j.clae.2007.01.002.

Rezeck, A. C., Mota, G. A., de Castro, P. N., \& Neves, A. C. C. (2020). Manual de cuidados e higienização para usuários de prótese ocular. Brazilian Journal of Health Review, 3(2), 3577-3584. https://doi.org/10.34119/bjhrv3n2-186.

Rodrigues, R. G. S., Rodrigues, D. S., \& de Oliveira, D. C. (2019). Reabilitação com prótese bucomaxilofacial: revisão de literatura. Revista Saúde Multidisciplinar, 5(1).

Rokohl, A. C., Koch, K. R., Trester, M., Trester, W., Pine, K. R., \& Heindl, L. M. (2018). Concerns of anophthalmic patients wearing cryolite glass prosthetic eyes. Ophthalmic Plastic \& Reconstructive Surgery, 34(4), 369-374. . https://10.1097/IOP.0000000000001021.

Souza, M. S. D. (2018). Prevalência microbiana das infecções oculares em pacientes atendidos no Hospital Universitário Onofre Lopes (HUOL-UFRN). Dissertação de Mestrado. Repositório Institucional.

Sperb, L. C. M., Neves, A. C. C., \& Rode, S. D. M. (2001). Considerações sobre prótese ocular-Sua importância na Odontologia atual. RGO (Porto Alegre), 49 (4), 202-204.

Tasso, C. O. (2019). Efetividade clínica de sabonetes líquidos desinfetantes no controle do biofilme presente em próteses totais removíveis. Tese de Doutorado. Repositório Institucional UNESP.

Tavares, C. C., Freire, J. C. P., Freire, S. C. P., Dias-Ribeiro, E., \& Batista, A. U. D. (2018). Aplicabilidade dos sistemas CAD/CAM em Prótese Total: revisão de literatura. Arch. Health Invest, 7 (11), 482-485. http://dx.doi.org/10.21270/archi.v7i11.3030.

Toribio, A., Marrodán, T., Fernández-Natal, I., Martínez-Blanco, H., Rodríguez-Aparicio, L., \& Ferrero, M. Á. (2017). Study of conjunctival flora in anophthalmic patients: influence on the comfort of the socket. Graefe's Archive for Clinical and Experimental Ophthalmology, 255(8), 1669-1679. https://doi.org/10.1007/s00417-017-3708-8.

Tsui, C., Kong, E. F., \& Jabra-Rizk, M. A. (2016). Pathogenesis of Candida albicans biofilm. FEMS Pathogens and Disease,74(4), 1018. https://doi.org/10.1093/femspd/ftw018.

Zanelatto, C. (2015). Ecologia de leveduras da cavidade bucal de pessoas saudáveis: diversidade de espécies e distribuição espacial. Dissertação de Mestrado. Repositório Institucional.

Zhang, Y., Zhang, M. N., Wang, X., \& Chen, X. F. (2015). Removal of the eye in a tertiary care center of China: a retrospective study on 573 cases in 20 years. International Journal of Ophthalmology, 8(5), 1024. https://doi.org/10.3980/j.issn.2222-3959.2015.05.3z. 